\title{
BcHTT4 Inhibits Branching of Non-Heading Chinese Cabbage at the Vegetative Stage
}

\author{
Mingliang Guo ${ }^{1,2,3}$, Lanlan Xu ${ }^{1,2,3}$, Yan Long ${ }^{1,2,3}$, Feiyi Huang 1,2,3, Tongkun Liu 1,2,3, Ying Li 1,2,3 \\ and Xilin Hou 1,2,3,* \\ 1 State Key Laboratory of Crop Genetics and Germplasm Enhancement, Ministry of Agriculture, \\ Nanjing 210095, China; 2017204019@njau.edu.cn (M.G.); 2019104059@njau.edu.cn (L.X.); \\ 2018104057@njau.edu.cn (Y.L.); hfy@njau.edu.cn (F.H.); liutk@njau.edu.cn (T.L.); yingli@njau.edu.cn (Y.L.) \\ 2 College of Horticulture, Nanjing Agricultural University, Nanjing 210095, China \\ 3 Key Laboratory of Southern Vegetable Crop Genetic Improvement, Ministry of Agriculture, \\ Nanjing 210095, China \\ * Correspondence: hxl@njau.edu.cn; Tel.: +86-25-8439-5756
}

check for updates

Citation: Guo, M.; Xu, L.; Long, Y.; Huang, F.; Liu, T.; Li, Y.; Hou, X. BcHTT4 Inhibits Branching of Non-Heading Chinese Cabbage at the Vegetative Stage. Plants 2021, 10, 510. https://doi.org/10.3390/plants 10030510

Academic Editor: Bertrand Dubreucq

Received: 27 January 2021

Accepted: 3 March 2021

Published: 9 March 2021

Publisher's Note: MDPI stays neutral with regard to jurisdictional claims in published maps and institutional affiliations.

Copyright: (c) 2021 by the authors. Licensee MDPI, Basel, Switzerland. This article is an open access article distributed under the terms and conditions of the Creative Commons Attribution (CC BY) license (https:/ / creativecommons.org/licenses/by/ $4.0 /)$.

\begin{abstract}
Branching is speculated to contribute to the plant architecture and crop yield. As a quantitative trait, branching is regulated by multiple genes in non-heading Chinese cabbage (NHCC). Several related candidate genes have been discovered in previous studies on the branching of NHCC, but their specific functions and regulatory mechanisms still need to be verified and explored. In this study, we found that the expression of BcHTT4, the ortholog to HEAT-INDUCED TAS1 TARGET4 (HTT4) in Arabidopsis, was significantly different between 'Suzhouqing' (common type) and 'Maertou' (multiple shoot branching type) in NHCC, which was consistent with the previous transcriptome sequencing results. The silencing of BcHTT4 expression in non-heading Chinese cabbage promotes axillary bud growth at the vegetative stage. When BcHTT4 is overexpressed in Arabidopsis, branching will decrease. In further study, we found that BcHTT4 interacts with immunophilin BcFKBP13 in vivo and in vitro through yeast two-hybrid analysis and bimolecular fluorescence complementation (BiFC) assays. Moreover, quantitative real-time PCR analysis showed that when the expression of BcHTT4 was silenced in 'Suzhouqing', the expression of BCFKBP13 also decreased significantly. Our findings reveal that $B C H T T 4$ is involved in the branching mechanism and interacts with immunophilin $B c F K B P 13$ in NHCC.
\end{abstract}

Keywords: branching; non-heading Chinese cabbage; virus-induced gene silencing; BcHTT4; FKBP13

\section{Introduction}

With the increasing population in the world, the problem of food production is becoming more and more prominent and needs to be solved urgently. The appropriate number of branches and tillers plays a decisive role in the yield of most crops [1-3]; thus, the research on branching and tillering has received more and more attention. As a leafy vegetable, the yield of non-heading Chinese cabbage (NHCC) is mainly determined by the number of leaves. The research on the branching of NHCC not only reveals the branching mechanism from the molecular level, but also helps to improve varieties to achieve the purpose of increasing yield. However, the branching regulation of NHCC is rarely reported.

Previous studies have shown that the whole branching process was composed of axillary bud formation and subsequent growth $[4,5]$. In rice, sorghum, Arabidopsis and other plants, researchers found that tillering is affected by the interaction of developmental factors [1,6,7], hormones [8-10] and environmental factors [11,12]. Environmental factors, including light, temperature, water, and nutrition, form a complex network that affects the growth and development of branching. Moreover, studies have shown that the photosynthetic activity and sugar utilization rate of buds also affect the branching to a certain extent $[13,14]$. 
Previously, many genes regulating branching have been found, such as MONOCULM 1 (MOC1) $[4,15,16]$ and DWARF27 (D27) [17] in rice, LATERAL SUPPRESSOR (LAS) $[18,19]$ and AUXIN RESISTANT 1 (AXR1) [20,21] in Arabidopsis, TEOSINTE BRANCHED 1 (TB1) [1,22] in maize, and so on. As a popular and widely grown vegetable, the research on the branching of NHCC has made some progress. The NHCC, which originated from China, is divided into five varieties: var. communis (Tsen et Lee) Hanelt, var. rosularis (Tsen et Lee) Hanelt, var. parachinensis (L.H. Bailey) Hanelt, var. tai-tsai Hort and var. multiceps Hort [23]. Compared with the other four varieties, var. multiceps Hort has unique growth characteristics and a large number of branches are formed at the vegetative stage. However, the axillary buds of other four varieties did not begin outgrowth until the reproductive stage, which was significantly different from that of the var. multiceps Hort. Therefore, var. multiceps Hort provides an ideal material for the study of branching mechanisms of NHCC at the vegetative stage.

In previous transcriptome sequencing analysis of mixed tissues, including roots, stems, leaves, flowers, and pods of the five varieties of NHCC, we found that the expression of BcHTT4, the ortholog to HEAT-INDUCED TAS1 TARGET4 (HTT4) in Arabidopsis, in var. multiceps Hort, was significantly different from that of the other four varieties. The expression of BcHTT4 was down-regulated in var. multiceps Hort, but up-regulated in the other four varieties. This suggests that BcHTT4 may participate in the branching regulation of NHCC to some extent, but this needs further verification. Virus-induced gene silencing (VIGS) is a kind of technology which can silence the target gene at transcription level after infecting the plant with a virus carrying the specific fragment of the target gene [24-26]. VIGS technology is an important tool in the study of gene function in plants. VIGS technology has been gradually used in gene function verification of NHCC, which provides a technical means for exploring the function of BcHTT4.

In Arabidopsis, HTT4 is the target of trans-acting small interfering RNAs (ta-siRNAs), which is a unique class of small, interfering plant RNAs (siRNAs) [27,28]. The expression of HTT4 is inhibited at the transcriptional level by complementing the sequences of target genes of siRNAs [29-31]. In addition, the expression of HTT4 was up-regulated at low temperature treatment [32] in Arabidopsis. However, as the target of ta-siRNAs, HTT4 encodes unknown functional proteins in Arabidopsis [33,34], and it has rarely been studied in other species. At present, it has been found that the expression of targets is silenced by small RNAs to regulate tillering. For example, the expression of rice SQUAMOSA promotor-binding protein-like (OsSPL14) gene is inhibited by microRNAs [35,36] to regulate tillering $[37,38]$.

Here, the expression of BcHTT4 showed opposite trends in 'Suzhouqing' and 'Maertou', which was analyzed by quantitative real-time PCR. In the functional analysis, we found that BcHTT4 plays a negative role in branching through utilizing VIGS technology in 'Suzhouqing' and overexpression in Arabidopsis. The results of yeast two-hybrid analysis and bimolecular fluorescence complementation assays showed that BcHTT4 interacts with $B c F K B P 13$ in vivo and in vitro. When the expression of HTT4 was silenced in 'Suzhouqing', the expression of $F K B P 13$ was also down-regulated.

\section{Results}

\subsection{BcHTT4 May Be a Negative Regulator of Branching in NHCC}

According to the previous transcriptome data of mixed tissues from five varieties of NHCC, the expression of BcHTT4 in var. multiceps Hort was significantly lower than that in the other four varieties. The unique branching characteristics of var. multiceps Hort at the vegetative stage and the difference of transcript level of BcHTT4 between var. multiceps Hort and the other four varieties indicated that $B c H T T 4$ was related to the branching of NHCC. To further explore the expression pattern of BcHTT4 in NHCC, we took samples at four periods of 'Suzhouqing' and 'Maertou' according to their growth characteristics and carried out quantitative real-time PCR. The quantitative real-time PCR analysis (Figure 1) showed that although BcHTT4 was expressed in all the samples tested 
from 'Suzhouqing' and 'Maertou', its expression in 'Suzhouqing' was significantly higher than that in 'Maertou', which is consistent with transcriptome sequencing data. Moreover, the expression of BcHTT4 in 'Suzhouqing' was significantly up-regulated with the plant growth, and the expression level reached the peak at 80 days after germination. Meanwhile, 'Maertou' was at the axillary bud outgrowth stage, but the expression pattern of BCHTT4 in 'Maertou' was the opposite from that in 'Suzhouqing'. The expression patterns of BcHTT4 in 'Suzhouqing' and 'Maertou' suggest that it seems to be an inhibitor of branching in NHCC.

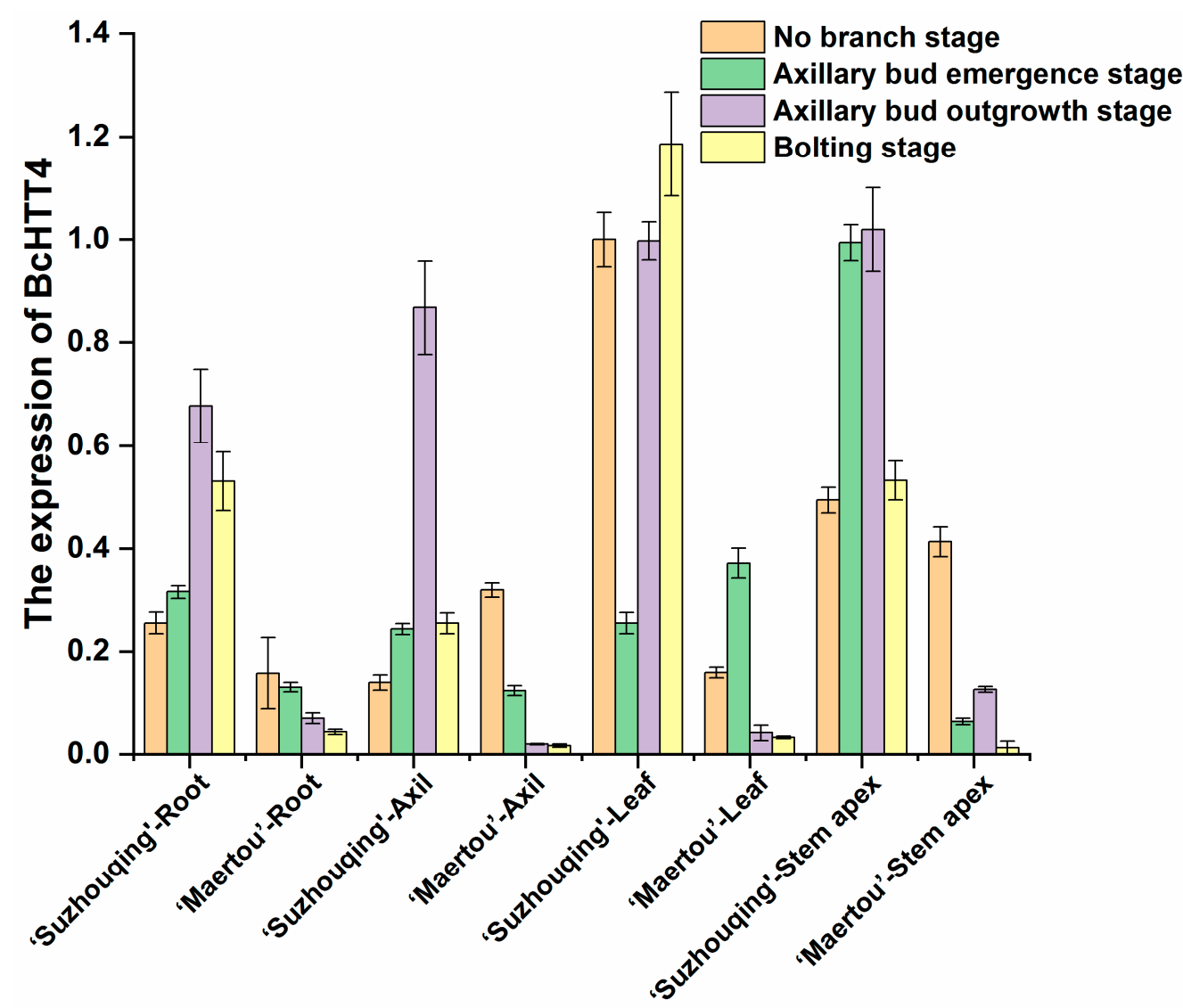

Figure 1. The transcription level of BcHTT4 in the two cultivars. No branch stage (at the 40th day after germination): no obvious axillary buds appeared at the five-leaf stage in two cultivars; axillary bud emergence stage (at the 49th day after germination): apparent axillary bud just occurred in 'Maertou' but it is still not in 'Suzhouqing'; axillary bud outgrowth stage (at the 80th day after germination): $70 \%$ axillary buds grown into branches in 'Maertou'; bolting stage (at the 105th day after germination): there are flower buds generated in two cultivars [23].

\subsection{The Characteristics of BcHTT4}

To verify whether the sequence of BcHTT4 is different in 'Suzhouqing' and 'Maertou', the coding and promoter sequence of BcHTT4 were amplified using specific primers (Table S1). Sequence alignment showed that there was no difference in the coding sequence of BcHTT4 between the two cultivars (Figure S1A). However, the promoter sequence of $B c H T T 4$ was significantly different between the two cultivars. Sequence alignment showed that there was a $65 \mathrm{bp}$ insertion in the BcHTT4 promoter of the 'Suzhouqing' of region approximately 660 bp upstream of the start codon (Figures 2A and S1B), which may have caused the expression difference of BcHTT4 between the two cultivars. Moreover, BcHTT4GFP fusions were localized in the cytoplasm and nucleus in Arabidopsis. We integrated GFP into the C-terminus of BcHTT4 and transiently expressed it in tobacco cells. The result of subcellular localization showed that BcHTT4-GFP fusions were localized in the cell cytoplasm (Figure 2B). 
A

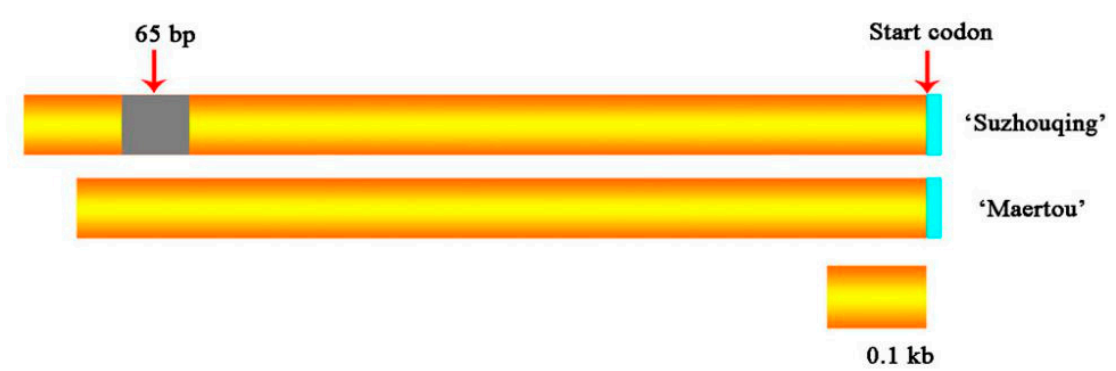

B

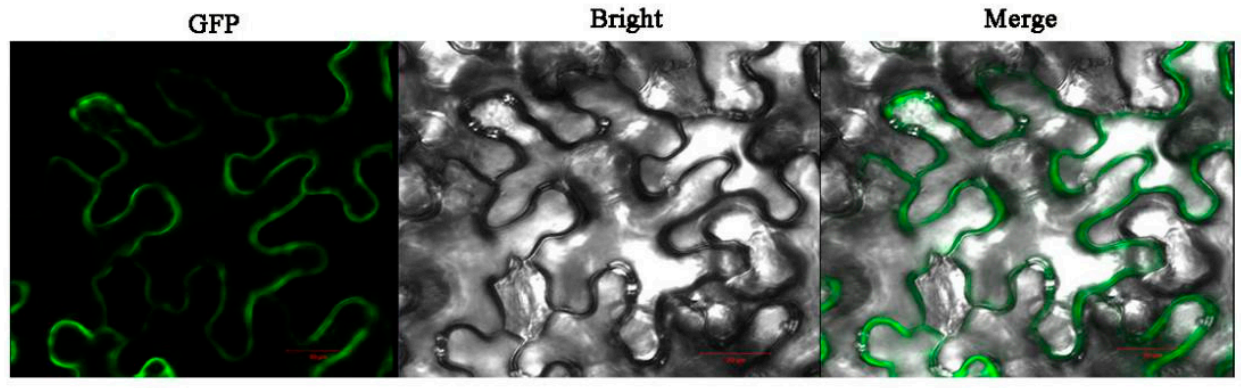

Figure 2. The characteristics of BcHTT4. (A) Alignment of promoter sequence of BcHTT4 in 'Suzhouqing' and 'Maertou'. (B) Subcellular localization of BcHTT4-GFP fusions in tobacco cells. The green fluorescence signal is displayed in the dark field and bright field and merged. Scale bar, $20 \mu \mathrm{m}$.

\subsection{Silencing of the BcHTT4 Expression Promotes Branching of 'Suzhouqing'}

The significant difference of transcription level and expression trend of BcHTT4 between 'Suzhouqing' and 'Maertou' indicated that BcHTT4 is a negative regulator in branching at the vegetative stage. 'Suzhouqing' is the representative of common cultivars; therefore, we used VIGS technology based on turnip yellow mosaic virus (TYMV) to silence the expression of BcHTT4 in 'Suzhouqing', so as to explore the function of BcHTT4 in branching at the vegetative stage. The pTY-BcHTT4 plasmids carrying the BcHTT4 specific fragment were used to infect 'Suzhouqing' plants utilizing particle bombardment at 25 days after germination. At the same time, 'Suzhouqing' plants were also infected by pTY-S plasmids. After 30 days of infection, the infected plants with obvious disease characteristics were obtained (Figure 3A). Through the observation of the leaf axils, we found that there were obvious axillary buds in 'Suzhouqing' infected by pTY-BcHTT4 plasmids, but there was no axillary bud in 'Suzhouqing' which grew naturally and was infected by pTY-S plasmids (Figure 3B). Through further quantitative real-time PCR analysis, we found that the expression of BcHTT4 decreased significantly in the plants infected with pTY-BcHTT4 plasmids, but there was no difference in the expression of BcHTT4 between plants which grew naturally, and plants infected by pTY-S plasmids (Figure 3C). These results showed that the silencing of BcHTT4 promotes branching in 'Suzhouqing' at the vegetative stage.

\subsection{Overexpression of BcHTT4 in Arabidopsis Resulted in Early Flowering and Branching Inhibition}

By silencing the expression of BcHTT4 in 'Suzhouqing', we conclude that it plays a negative role in the branching regulation of NHCC. In order to further verify its function, we obtained BcHTT4 overexpression Arabidopsis lines (Figure 4A). We found that the branches of transgenic Arabidopsis lines decreased sharply, which was opposite to the results of silencing BcHTT4 in 'Suzhouqing' (Figure 4D). In addition, BcHTT4-overexpression transgenic lines showed earlier bolting and flowering, which was about six days earlier than that of wild type Arabidopsis (Figure 4B,C). 

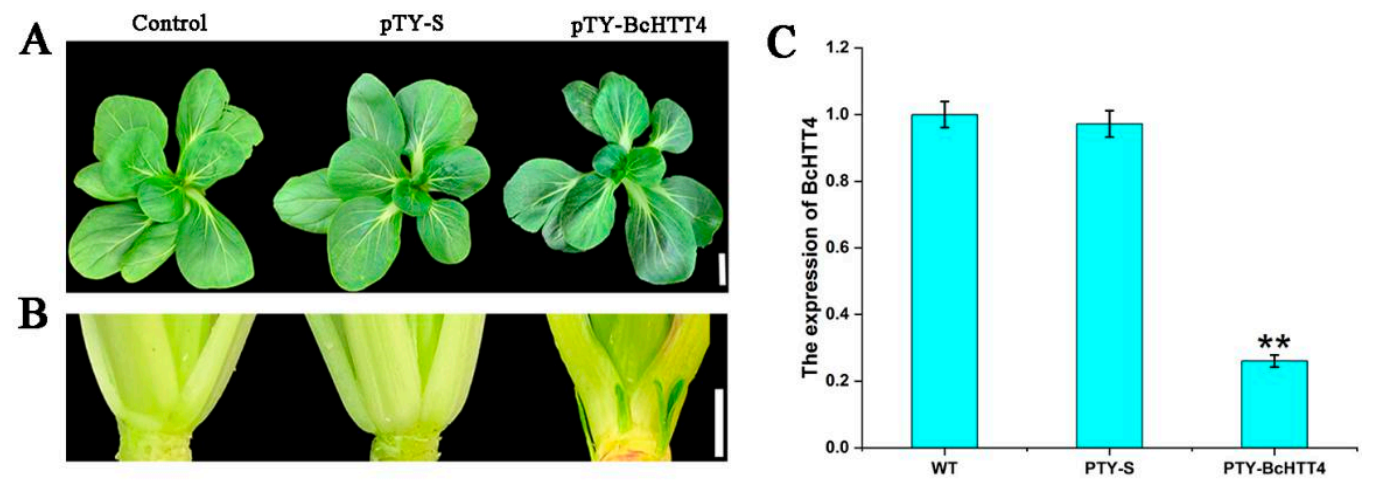

Figure 3. Silencing the expression of BcHTT4 in 'Suzhouqing'. (A) Phenotypes of 'Suzhouqing': uninfected, 'Suzhouqing' infected with pTY-S plasmids, and 'Suzhouqing' treated with pTY-BcHTT4 plasmids. Scale bar, $2 \mathrm{~cm}$. (B) Comparison of leaf axils among 'Suzhouqing': uninfected, 'Suzhouqing' infected with pTY-S plasmids, and 'Suzhouqing' infected with pTY-BcHTT4 plasmids in plant, leaf and axil. Scale bar, $1 \mathrm{~cm}$. (C) Transcription level of BcHTT4 under three different treatments of 'Suzhouqing'. Bars with double asterisks are significantly different at $p<0.05$, according to Tukey's single factor tests. Data are shown as the means \pm SD.

A
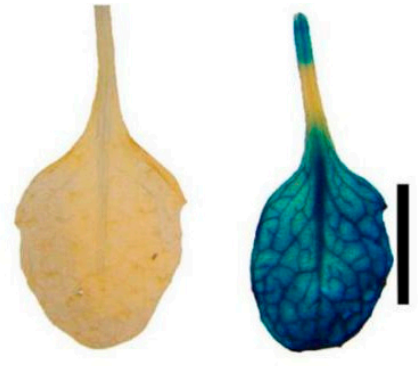

B

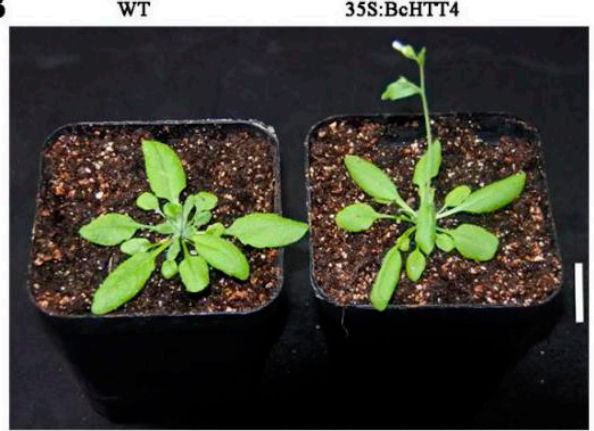

C

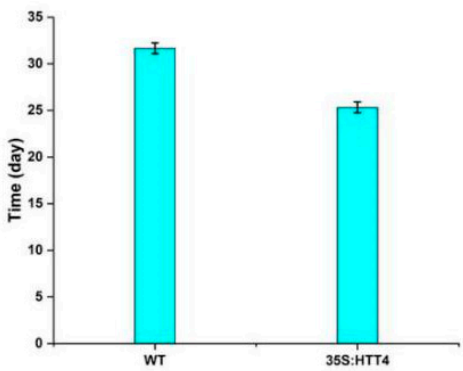

D

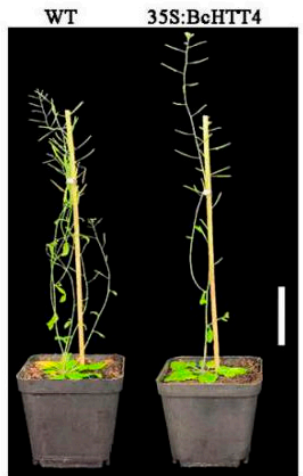

Figure 4. Overexpression of BcHTT4 in Arabidopsis. (A) The $\beta$-glucuronidase (Gus) staining of Arabidopsis. Scale bar, $5 \mathrm{~mm}$. (B,C) Comparison of bolting time between transgenic Arabidopsis lines and wild type Arabidopsis. (B) Scale bar, $2 \mathrm{~cm}$. (D) Observation on branch phenotype in transgenic Arabidopsis lines and wild type Arabidopsis. Scale bar, $5 \mathrm{~cm}$.

\subsection{BcHTT4 Interacts with BcFKBP13}

In plants, the study of HTT4 interacting protein is rare. To explore the regulatory mechanism of BcHTT4 in NHCC, we carried out a screening library (total mRNA) by yeast two-hybrid assay. Through the screening library, we found that a gene, which was the ortholog to AtFKBP13, interacts with BcHTT4 and was named BcFKBP13. The results of one-to-one verification of the yeast two-hybrid assay showed that BcHTT4 interacts with $B c F K B P 13$ (Figure 5A). In addition, the yellow fluorescence in co-transformed tobacco cells expressing BcHTT4-cYFP and BcFKBP13-nYFP indicated that BcHTT4 interacts with $B c F K B P 13$ in vivo through $\mathrm{BiFC}$ assays (Figure $5 \mathrm{~B}$ ). Taken together, these results indicate 
that $B c H T T 4$ interacts with $B c F K B P 13$ in vivo and in vitro. To further explore the interaction between $B c H T T 4$ and $B c F K B P 13$, we obtained the expression level of $B c F K B P 13$ when the expression of BcHTT4 was silenced in 'Suzhouqing' using quantitative real-time PCR. We found that when the expression of BcHTT4 was silenced, the expression of BcFKBP13 also decreased significantly (Figure 5C).

A
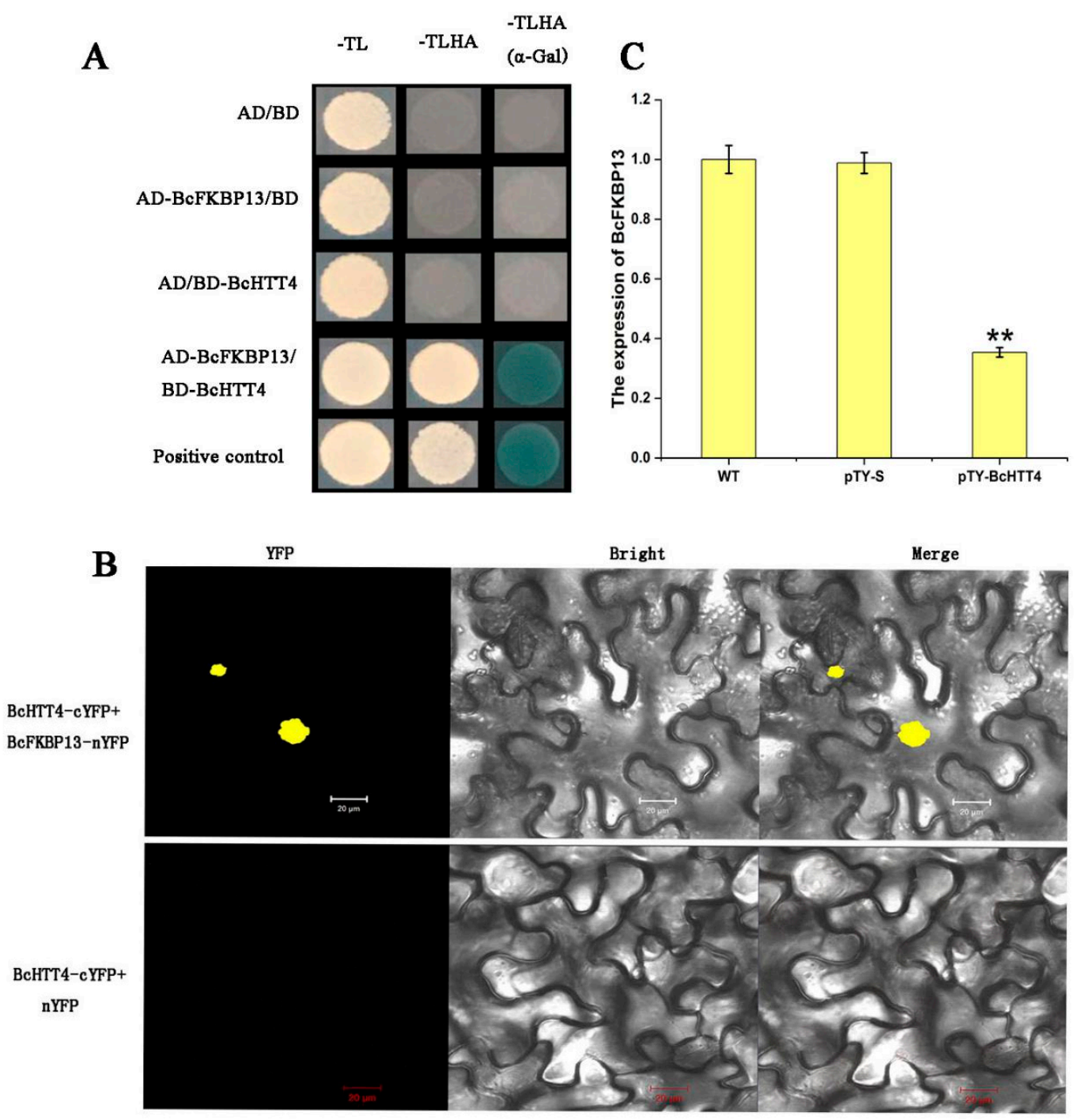

Figure 5. The interaction between BcHTT4 and BcFKBP13. (A) The verification of the interaction between $B c H T T 4$ and $B C F K B P 13$ by yeast two-hybrid assay. The yeast cells were grown on the SD media: SD-TL (left), SD-TLHA (middle), SD-TLHA (added $\alpha$-Gal, right). (B) Bimolecular fluorescence complementation (BiFC) analysis of the interaction between BcHTT4 and BcFKBP13. Yellow fluorescence signal is displayed in a dark field and bright field, respectively, and merged. Scale bar, $20 \mu \mathrm{m}$. (C) Transcription level of BcFKBP13 under three different treatments of 'Suzhouqing'. Bars with double asterisks are significantly different at $p<0.05$, according to Tukey's single factor tests. Data are shown as the means $\pm \mathrm{SD}$.

\section{Discussion}

Branching is an important agronomic character of plants, especially its function in yield [3,39]. Although many important genes related to branching have been found $[4,18,40]$, there are still a lot of genes regulating branching that have not yet been discovered. Among the factors affecting branching, temperature and photosynthesis are also related to branching $[13,14]$. In the research of NHCC, the regulation mechanism of branching development is still in its infancy, and the gene regulation involved in branching development is rare. 
Our discovery of BcHTT4 regulating branching development is a supplement to the study of branching development of NHCC.

In Arabidopsis, the expression of HTT4 is regulated by temperature. The expression of HTT4 decreased with the increase in temperature in Arabidopsis [30,41]. However, previous transcriptome data showed that there were significant differences in BcHTT4 transcription levels between the branching cultivars and common cultivars. In our research, the difference of HTT4 expression was further verified by quantitative real-time PCR in four parts, examined at four stages of 'Suzhouqing' and 'Maertou'. Our study found that the transcription level of BcHTT4 was significantly different between 'Suzhouqing' and 'Maertou', and with the occurrence and development of axillary buds, the expression of BcHTT4 gradually increased in 'Suzhouqing' while the expression of BcHTT4 showed a downward trend in 'Maertou', suggesting a putative negative regulator in the branching development of NHCC. Moreover, the difference of BcHTT4 promoter between 'Suzhouqing' and 'Maertou' may be responsible for the difference of expression.

In Arabidopsis, the function of HTT4 is not clear. In our study, we found that when the expression of BcHTT4 was silenced in 'Suzhouqing', it promoted the branching of 'Suzhouqing' at the vegetative stage, and when BcHTT4 was overexpressed in Arabidopsis, the number of branches was significantly reduced. These results indicated that BcHTT4 inhibits branching. Branching has a profound effect on the yield of NHCC; improving the branching quality of NHCC is an important way to increase yield. The function of BcHTT4 in the regulation of NHCC branching and the difference of the promoter sequence provide a theoretical basis for the yield increase in NHCC in the future. BcHTT4 plays a negative role in the branching regulation of NHCC; thus, we can down regulate the expression of BcHTT4 and even lose the BcHTT4 gene function to improve the branching of NHCC. In addition, the expression of HTT4 is closely related to temperature in Arabidopsis [30,41], which suggests that branching of NHCC could be affected by temperature.

Immunophilins [42,43] are found in many living cells. According to the difference of receptors, these immunophilins can be divided into two categories: cyclophilins and FKBPs (FK506 binding proteins). These receptor proteins are widely involved in many life processes [44-46]. In Arabidopsis, FKBP13, as an immunophilin of chloroplast thylakoid lumen [47], is involved in the transport of Rieske protein and regulates the level of Rieske protein in chloroplast thylakoid lumen through the precursor of FKBP13 interacting with the Rieske subunit [48]. The Rieske subunit, as a part of cytochrome bf complex, plays an important role in photosynthesis. Our study showed that BcHTT4 interacts with BcFKBP13 by a yeast two-hybrid assay and BiFC assay. In the 'Suzhouqing' plants infected by pTY$B c H T T 4$ plasmids, the expression of BcFKBP13 decreased with the silencing of BcHTT4 expression, which suggested that $B c H T T 4$ might promote the expression of $B c F K B P 13$ and affect the accumulation of Rieske protein.

In conclusion, we infer that $B C H T T 4$ acts as a negative regulator in the branching of NHCC at the vegetative stage, and that BcHTT4 interacts with BcFKBP13 in NHCC. Moreover, the precursor of $F K B P 13$ binds to Rieske protein and negatively regulates the accumulation of Rieske protein in Arabidopsis. Rieske protein belongs to the subunit of cytochrome bf complex and participates in the photosynthesis of plants. Therefore, we infer that BcHTT4 may regulate the branching of NHCC at the vegetative stage by affecting photosynthesis; however, this needs to be further verified.

\section{Materials and Methods}

\subsection{Plant Materials and Growth Conditions}

NHCC materials included two cultivars: B. rapa ssp. chinensis var. communis Tesnet Lee, cv. 'Suzhouqing' (common type) and B. rapa ssp. chinensis var. multiceps, cv. 'Maertou' (multiple shoot branching type). The branching characters of these two materials are obviously different in the vegetative stage. Both cultivars were planted in a greenhouse under a light-dark cycle with $16 \mathrm{~h}$ light and $8 \mathrm{~h}$ dark at $25^{\circ} \mathrm{C}$. 


\subsection{Tissue Sampling of 'Suzhouqing' and 'Maertou'}

The roots, stem apexes, leaves and leaf axils of the two materials were sampled in four stages according to the growth and development process of the two cultivars: the 5-leaf stage in two cultivars (no branch stage); apparent axillary bud just occurred in 'Maertou' but it had still not in 'Suzhouqing' (axillary buds emergence stage); 70\% axillary buds had grown into branches in 'Maertou' (axillary buds outgrowth stage); there are flower buds generated in two cultivars (bolting stage). RNA was then extracted and qPCR was conducted as below.

\subsection{Quantitative Real-Time PCR}

Total RNAs from sampled tissues were isolated using an RNeasy Plant Mini Kit (Qiagen) according to the user's manual. First strand cDNA was synthesized from $5 \mu \mathrm{g}$ of total RNA using the PrimeScript First-Strand cDNA Synthesis kit (TaKaRa, Dalian, China), according to the manufacturer's instructions. Real-time PCR experiments were performed using a QuantiTect SYBR Green real-time-PCR kit (TaKaRa, Dalian, China) in accordance with the manufacturer's protocols. The BcHTT4-specific primers QBcHTT4-F and QBcHTT4-R (Table S1) were designed using Beacon Designer 7.0 software, and $\beta$ actin (Table S1) was selected as the internal control gene. The relative expression levels of the selected transcripts were normalized to $\beta$-actin gene and calculated using the $2^{-\Delta \Delta \mathrm{Ct}}$ method.

\subsection{Acquisition of BcHTT4 Sequence}

The promoter and coding sequences of BcHTT4 in 'Suzhouqing' and 'Maertou' were amplified using specific primers (Table S1) and cloned into pEASY-Blunt Zero Cloning Vector (TranGen Biotech, China) for sequencing. Sequence alignments were performed using DNAMAN 6.0 software.

\subsection{Subcellular Localization of BcHTT4-GFP Fusions}

To construct the BcHTT4-GFP fusions, the coding sequence of BcHTT4 was amplified by primers BcHTT4-NdeI-F and BcHTT4-KpnI-R (Table S1) and cloned into pRI101 vector plasmids. The BcHTT4-GFP plasmids were transformed into $A$. tumefaciens strain GV3101. The transformed agrobacterium strains were infected into tobacco leaves when agrobacterium cells were cultured in yeast extract broth liquid medium until the OD600 reached approximately 0.8 . The infected tobacco leaves were observed under an Olympus Fluoview1000 microscope after 2-3 days of darkness [41].

\subsection{Silencing BcHTT4 Expression by VIGS Technology}

VIGS [24-26] technology carrying TYMV was used to study the function of BcHTT4. The $80 \mathrm{nt}$ palindromic oligonucleotide sequence specific to $B c H T T 4$ named pTY-BcHTT4 (Table S2) was fused into pTY-S plasmids to construct pTY-BcHTT4 plasmids and were used to infect 'Suzhouqing' plants, avoiding affecting the expression of other ortholog genes. The amplification of pTY-BcHTT4 plasmids of the expected size (522 nt) was used to identify positive clones by TYMV-specific primers PTYMV-F and pTYMV-R (Table S1). To infect 'Suzhouqing' with pTY-BcHTT4 plasmids, $5 \mu \mathrm{g}$ purified pTY-BcHTT4 plasmids

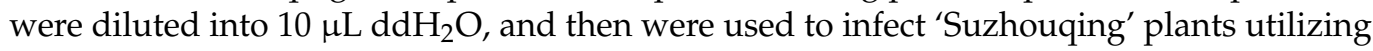
particle bombardment. The 'Suzhouqing' plants that were infiltrated with pTY-S vector plasmids were regarded as controls.

\subsection{Arabidopsis Transgenic Vector Construction and Transformation}

To construction BcHTT4 overexpression plasmids, we amplified the full-length coding sequence of BcHTT4 with primers BcHTT4-clone-F and BcHTT4-clone-R (Table S1) and cloned it into plant overexpression vector pTCK303 using primers BcHTT4-BamHI-F and BcHTT4-SpeI-R (Table S1). Transgenic vector plasmids were transformed into A. tumefaciens strain GV3101, and then were cultured in yeast extract broth (YEB) liquid medium until 
OD600 $=1.8$. The transgenic experiments were conducted through agrobacterium-mediated Arabidopsis transformation (floral dip) [49].

\subsection{The GUS Staining}

The X-gluc buffer was prepared and consisted of $100 \mathrm{mM}$ sodium phosphate, $\mathrm{pH}$ 7.0, $1 \mathrm{mM}$ potassium ferricyanide, $1 \mathrm{mM}$ potassium ferrocyanide, $10 \mathrm{mM}$ Na2-EDTA, $0.5 \% \mathrm{v} / \mathrm{v}$ Triton X-100, 20\% v/v methanol, and $0.5 \mathrm{mg} / \mathrm{mL} \mathrm{X-gluc.} \mathrm{Leaves} \mathrm{from} \mathrm{transgenic} \mathrm{and}$ wild type plants were incubated in X-gluc buffer at $37^{\circ} \mathrm{C}$ for $12 \mathrm{~h}$. Then, the leaves were immersed in $75 \%$ ethanol, incubated at room temperature to remove the chlorophyll, and photographed.

\subsection{Yeast Two-Hybrid Assay}

The coding sequences of BcHTT4 that was amplified by primers were named BcHTT4NdeI-BD-F and BcHTT4-EcoRI-BD-R (Table S1) and cloned into pGBKT7 vector to generate $\mathrm{BD}-B c H T T 4$ fusions. The coding sequence of $B c F K B P 13$ was amplified by primers named $B c F K B P 13-N d e I-A D-F$ and BcFKBP13-ClaI-AD-R (Table S1) and cloned into pGADT7 vector to generate $\mathrm{AD}-B c F K B P 13$ fusions. The fusion constructs, negative control plasmids $\mathrm{AD}$ (activation domain) and $\mathrm{BD}$ (binding domain), and positive control plasmids pGBKT7-53 DNA-BD and pGADT7-T were transformed into Golden Yeast (Clontech, China) cells through the lithium acetate-mediated method. The transformed yeast strains were grown on SD/-Trp-Leu (Clontech, China) medium, SD/-Leu-Trp-His-Ade (Clontech, China) medium without $\alpha$-Gal, and SD/-Leu-Trp-His- Ade (Clontech, China) medium, respectively, with $\alpha$-Gal at $28{ }^{\circ} \mathrm{C}$ for $2-3$ days.

\subsection{BiFC Assays in Tobacco}

The coding sequence of BcHTT4 was amplified by primers BcHTT4-XbaI-cYFP-F and $B c H T T 4-K p n I-c Y F P-R$ (Table S1) and cloned into pUC-SPYCE vector to generate BcHTT4cYFP plasmids. The coding sequence of $B c F K B P 13$ was amplified by primers $B c F K B P 13-$ XbaI-cYFP-F and BcFKBP13-KpnI-cYFP-R (Table S1) and cloned into pUC-SPYNE for generating $B c F K B P 13-n Y F P$. The plasmid mixtures (BcHTT4-cYFP and BcFKBP13-nYFP, $B c H T T 4$-cYFP and pUC-SPYNE) were introduced into the tobacco cells. After incubation in the dark for $36 \mathrm{~h}$, the yellow fluorescence signal was observed using an Olympus Fluoview1000 microscope [50].

Supplementary Materials: The following are available online at https://www.mdpi.com/2223-774 7/10/3/510/s1: Table S1. The sequence of all the primers; Table S2. Sequences for construction of the VIGS vector; Figure S1. Alignment of coding region (A), and promoter sequence (B) of BcHTT4 from 'Suzhouqing' and 'Maertou'.

Author Contributions: Conceptualization, M.G. and L.X.; methodology, M.G.; software, M.G.; validation, L.X.; formal analysis, Y.L. (Yan Long); investigation, F.H.; resources, T.L.; data curation, M.G.; writing—original draft preparation, M.G.; writing—review and editing, M.G.; visualization, Y.L. (Ying Li); supervision, Y.L. (Ying Li); project administration, X.H.; funding acquisition, X.H. All authors have read and agreed to the published version of the manuscript.

Funding: This study was supported by the National Natural Science Foundation of China (31872106), the National Key Research and Development Program (2018YFD1000800 and 2017YFD0101803), and the National Vegetable Industry Technology System (CARS-23-A-06).

Institutional Review Board Statement: Not applicable.

Informed Consent Statement: Not applicable.

Data Availability Statement: All the data are in the manuscript.

Conflicts of Interest: The authors declare no conflict of interest. 


\section{References}

1. Doebley, J.F.; Gaut, B.S.; Smith, B.D. The molecular genetics of crop domestication. Cell 2006, 127, 1309-1321. [CrossRef]

2. Doust, A.N.; Kellogg, E.A. Effect of genotype and environment on branching in weedy green millet (Setaria viridis) and domesticated foxtail millet (Setaria italica) (Poaceae). Mol. Ecol. 2006, 15, 1335-1349. [CrossRef] [PubMed]

3. Xing, Y.; Zhang, Q. Genetic and molecular bases of rice yield. Ann. Rev. Plant Biol. 2010, 61, 421-442. [CrossRef]

4. Li, X.; Qian, Q.; Fu, Z.; Wang, Y.; Xiong, G.; Zeng, D.; Wang, X.; Liu, X.; Teng, S.; Hiroshi, F.; et al. Control of tillering in rice. Nature 2003, 422, 618-621. [CrossRef] [PubMed]

5. Wang, B.; Smith, S.M.; Li, J. Genetic regulation of shoot architecture. Annu. Rev. Plant Biol. 2018, 69, 437-468. [CrossRef]

6. Stirnberg, P.; Furner, I.J.; Ottoline Leyser, H.M. MAX2 participates in an SCF complex which acts locally at the node to suppress shoot branching. Plant J. 2007, 50, 80-94. [CrossRef]

7. Booker, J.; Sieberer, T.; Wright, W.; Williamson, L.; Willett, B.; Stirnberg, P.; Turnbull, C.; Srinivasan, M.; Goddard, P.; Leyser, O. MAX1 encodes a cytochrome P450 family member that acts downstream of MAX3/4 to produce a carotenoid derived branch-inhibiting hormone. Dev. Cell 2005, 8, 443-449. [CrossRef]

8. Cai, T.; Meng, X.; Liu, X.; Liu, T.; Wang, H.; Jia, Z.; Yang, D.; Ren, X. Exogenous hormonal application regulates the occurrence of wheat tillers by changing endogenous hormones. Front. Plant Sci. 2018, 9, 1886. [CrossRef] [PubMed]

9. Morris, S.E.; Cox, M.C.; Ross, J.J.; Krisantini, S.; Beveridge, C.A. Auxin dynamics after decapitation are not correlated with the initial growth of axillary buds. Plant Physiol. 2005, 138, 1665-1672. [CrossRef]

10. Prusinkiewicz, P.; Crawford, S.; Smith, R.S.; Ljung, K.; Bennett, T.; Ongaro, V.; Leyser, O. Control of bud activation by an auxin transport switch. Proc. Nat. Acad. Sci. USA 2009, 106, 17431-17436. [CrossRef] [PubMed]

11. Kim, H.K.; van Oosterom, E.; Dingkuhn, M.; Luquet, D.; Hammer, G. Regulation of tillering in sorghum: Environmental effects. Ann. Bot. 2010, 106, 57-67. [CrossRef] [PubMed]

12. Leduc, N.; Roman, H.; Barbier, F.; Péron, T.; Huché-Thélier, L.; Lothier, J.; Demotes-Mainard, S.; Sakr, S. Light signaling in bud outgrowth and branching in plants. Plants 2014, 3, 223-250. [CrossRef]

13. Barbier, F.F.; Lunn, J.E.; Beveridge, C.A. Ready, steady, go! A sugar hit starts the race to shoot branching. Curr. Opin. Plant Biol. 2015, 25, 39-45. [CrossRef] [PubMed]

14. Evers, J.B. Sugar as a key component of the shoot branching regulation network. Plant Cell Environ. 2015, 38, 1455-1456. [CrossRef] [PubMed]

15. Liao, Z.; Yu, H.; Duan, J.; Yuan, K.; Yu, C.; Meng, X.; Kou, L.; Chen, M.; Jing, Y.; Liu, G.; et al. SLR1 inhibits MOC1 degradation to coordinate tiller number and plant height in rice. Nat. Commun. 2019, 10, 2738. [CrossRef] [PubMed]

16. Shao, G.; Lu, Z.; Xiong, J.; Wang, B.; Jing, Y.; Meng, X.; Liu, G.; Ma, H.; Liang, Y.; Chen, F.; et al. Tiller bud formation regulators MOC1 and MOC3 cooperatively promote tiller bud outgrowth by activating FON1 expression in rice. Mol. Plant 2019, 12, 1090-1102. [CrossRef] [PubMed]

17. Lin, H.; Wang, R.; Qian, Q.; Yan, M.; Meng, X.; Fu, Z.; Yan, C.; Jiang, B.; Su, Z.; Li, J.; et al. DWARF27, an iron-containing protein required for the biosynthesis of strigolactones, regulates rice tiller bud outgrowth. Plant Cell 2009, 21, 1512-1525. [CrossRef] [PubMed]

18. Greb, T.; Clarenz, O.; Schafer, E.; Muller, D.; Herrero, R.; Schmitz, G.; Theres, K. Molecular analysis of the LATERAL SUPPRESSOR gene in Arabidopsis reveals a conserved control mechanism for axillary meristem formation. Genes Dev. 2003, 17, 1175-1187. [CrossRef] [PubMed]

19. Yang, F.; Wang, Q.; Schmitz, G.; Müller, D.; Theres, K. The bHLH protein ROX acts in concert with RAX1 and LAS to modulate axillary meristem formation in Arabidopsis. Plant J. 2012, 71, 61-70. [CrossRef]

20. Stirnberg, P.; Chatfield, S.P.; Leyser, H.M. AXR1 acts after lateral bud formation to inhibit lateral bud growth in Arabidopsis. Plant Physiol. 1999, 121, 839-847. [CrossRef]

21. Stirnberg, P.; Zhao, S.; Williamson, L.; Ward, S.; Leyser, O. FHY3 promotes shoot branching and stress tolerance in Arabidopsis in an AXR1-dependent manner. Plant J. 2012, 71, 907-920. [CrossRef]

22. Takeda, T.; Suwa, Y.; Suzuki, M.; Kitano, H.; Ueguchi-Tanaka, M.; Ashikari, M.; Matsuoka, M.; Ueguchi, C. The OsTB1 gene negatively regulates lateral branching in rice. Plant J. 2003, 33, 513-520. [CrossRef]

23. Cao, X.; Cui, H.; Li, J.; Xiong, A.; Hou, X.; Li, Y. Heritability and gene effects for tiller number and leaf number in non-heading Chinese cabbage estimated by joint segregation analysis. Scientia Horticulturae 2016, 203, 199-206. [CrossRef]

24. Kumagai, M.H.; Donson, J.; della-Cioppa, G.; Harvey, D.; Hanley, K.; Grill, L.K. Cytoplasmic inhibition of carotenoid biosynthesis with virus-derived RNA. Proc. Natl. Acad. Sci. USA 1995, 92, 1679-1683. [CrossRef] [PubMed]

25. Baulcombe, D.C. RNA as a target and an initiator of posttranscriptional gene silencing in transgenic plants. Plant Mol. Biol. 1996, 32, 79-88. [CrossRef] [PubMed]

26. Baulcombe, D.C. Fast forward genetics based on virus induced gene silencing. Curr. Opin. Plant Biol. 1999, 2, 109-113. [CrossRef]

27. Ramachandran, V.; Chen, X. Small RNA metabolism in Arabidopsis. Trends Plant Sci. 2008, 13, 368-374. [CrossRef] [PubMed]

28. Carthew, R.W.; Sontheimer, E.J. Origins and mechanisms of miRNAs and siRNAs. Cell 2009, 136, 642-655. [CrossRef] [PubMed]

29. Doench, J.G.; Petersen, C.P.; Sharp, P.A. siRNAs can function as miRNAs. Genes Dev. 2003, 17, 438-442. [CrossRef] [PubMed]

30. Khvorova, A.; Reynolds, A.; Jayasena, S.D. Functional siRNAs and miRNAs exhibit strand bias. Cell 2003, 115, 209-216. [CrossRef]

31. Valencia-Sanchez, M.A.; Liu, J.; Hannon, G.J.; Parker, R. Control of translation and mRNA degradation by miRNAs and siRNAs. Genes Dev. 2006, 20, 515-524. [CrossRef] [PubMed] 
32. Kume, K.; Tsutsumi, K.; Saitoh, Y. TAS1 trans-acting siRNA targets are differentially regulated at low temperature, and TAS1 trans-acting siRNA mediates temperature-controlled At1g51670 expression. Biosci. Biotechnol. Biochem. 2010, 74, 1435-1440. [CrossRef]

33. Vazquez, F.; Vaucheret, H.; Rajagopalan, R.; Lepers, C.; Gasciolli, V.; Mallory, A.C.; Hilbert, J.L.; Bartel, D.P.; Crété, P. Endogenous trans-acting siRNAs regulate the accumulation of Arabidopsis mRNAs. Mol. Cell 2004, 16, 69-79. [CrossRef]

34. Allen, E.; Xie, Z.; Gustafson, A.M.; Carrington, J.C. microRNA-directed phasing during trans-acting siRNA biogenesis in plants. Cell 2005, 121, 207-221. [CrossRef] [PubMed]

35. Mourelatos, Z. miRNPs: A novel class of ribonucleoproteins containing numerous microRNAs. Genes Dev. 2002, 16, 720-728. [CrossRef]

36. Bartel, D.P. MicroRNAs: Genomics, biogenesis, mechanism, and function. Cell 2004, 116, 281-297. [CrossRef]

37. Rhoades, M.W.; Reinhart, B.J.; Lim, L.P.; Burge, C.B.; Bartel, B.; Bartel, D.P. Prediction of plant microRNA targets. Cell 2002, 110, 513-520. [CrossRef]

38. Xie, K.B.; Wu, C.; Xiong, L. Genomic organization, differential expression, and interaction of SQUAMOSA promoter-binding-like transcription factors and microRNA156 in rice. Plant Physiol. 2006, 14, 280-293. [CrossRef] [PubMed]

39. Jones, H.G.; Kirby, E.J.M. Effects of manipulation of number of tillers and water supply on grain yield in barley. J. Agric. Sci. 1977, 88, 391-397. [CrossRef]

40. Schumacher, K.; Schmitt, T.; Rossberg, M.; Schmitz, G.; Theres, K. The Lateral suppressor (Ls) gene of tomato encodes a new member of the VHIID protein family. Proc. Natl. Acad. Sci. USA 1999, 96, 290-295. [CrossRef] [PubMed]

41. Li, S.; Liu, J.; Liu, Z.; Li, X.; Wu, F.; He, Y. Heat-induced TAS1 target1 mediates thermotolerance via heat stress transcription factor A1a-directed pathways in Arabidopsis. Plant Cell 2014, 26, 1764-1780. [CrossRef] [PubMed]

42. Schreiber, S.L. Chemistry and biology of the immunophilins and their immunosuppressive ligands. Science 1991, 251, 283-287. [CrossRef]

43. Paić, A.T.; Fulgosi, H. Chloroplast immunophilins. Protoplasma 2016, 253, 249-258. [CrossRef] [PubMed]

44. Bailly, A.; Sovero, V.; Geisler, M. The twisted dwarf's ABC: How immunophilins regulate auxin transport. Plant Signal. Behav. 2006, 1, 277-280. [CrossRef] [PubMed]

45. Aviezer-Hagai, K.; Skovorodnikova, J.; Galigniana, M.; Farchi-Pisanty, O.; Maayan, E.; Bocovza, S.; Efrat, Y.; von Koskull-Döring, P.; Ohad, N.; Breiman, A. Arabidopsis immunophilins ROF1 (AtFKBP62) and ROF2 (AtFKBP65) exhibit tissue specificity, are heat-stress induced, and bind HSP90. Plant Mol. Biol. 2007, 63, 237-255. [CrossRef]

46. Wang, Y.; Liu, C.; Yang, D.; Yu, H.; Liou, Y.C. Pin1At encoding a peptidyl-prolyl cis/trans isomerase regulates flowering time in Arabidopsis. Mol. Cell 2010, 37, 112-122. [CrossRef] [PubMed]

47. Edvardsson, A.; Shapiguzov, A.; Petersson, U.A.; Schröder, W.P.; Vener, A.V. Immunophilin AtFKBP13 sustains all peptidyl-prolyl isomerase activity in the thylakoid lumen from Arabidopsis thaliana deficient in AtCYP20-2. Biochemistry 2007, 46, 9432-9442. [CrossRef]

48. Gupta, R.; Mould, R.M.; He, Z.; Luan, S. A chloroplast FKBP interacts with and affects the accumulation of Rieske subunit of cytochrome bf complex. Proc. Natl. Acad. Sci. USA 2002, 99, 15806-15811. [CrossRef] [PubMed]

49. Clough, S.J.; Bent, A.F. Floral dip: A simplified method for Agrobacterium-mediated transformation of Arabidopsis thaliana. Plant J. 1998, 16, 735-743. [CrossRef]

50. Niwa, M.; Daimon, Y.; Kurotani, K.; Higo, A.; Pruneda-Paz, J.L.; Breton, G.; Mitsuda, N.; Kay, S.A.; Ohme-Takagi, M.; Endo, M.; et al. BRANCHED1 interacts with FLOWERING LOCUS T to repress the floral transition of the axillary meristems in Arabidopsis. Plant Cell 2013, 25, 1228-1242. [CrossRef] [PubMed] 